me ist der Anbieter Zollsoft aus Jena, der mit „Tomedo“ eines der wenigen Praxissoftware-Pakete für Apple-Macintosh-Rechner liefert. „Da wir sowohl in der Medizin als auch in der Apple-Welt zuhause sind, war für uns von Anfang an klar, dass iPad und iPhone in der Konzeption unserer Software eine wichtige Rolle spielen“, berichtet Dr. Andreas Zollmann, der das Softwarehaus gemeinsam mit seinem Bruder Johannes gegründet hatte. So bot Tomedo fast von Anfang an die Option, digitale Patientenakten auf dem Tablet zu Hausbesuchen mitzunehmen oder mit den Apple-Mobilgeräten Befundbilder zu fotografieren und sie direkt in die Patientenakte zu übernehmen.

\section{Weniger fehleranfällig}

Seit kurzem bietet die Praxissoftware aus Jena nun auch Anamnesebögen und andere Digitalformulare auf dem iPad.
Unkelbach hat sie schon im Einsatz: „Den Patienten macht es Spaß und im täglichen Ablauf der Praxis spart das Tablet viel Zeit."So musste früher eine Medizinische Fachangestellte jede einzelne Frage im Versicherungsfragebogen mit dem Patienten durchgehen. Heute kann sie der Patient selbst Schritt für Schritt auf dem iPad lesen und beantworten. Sobald der Patient die Angaben im Formular bestätigt, landet dieses ohne weiteres Zutun des Praxispersonals in der digitalen Akte, wo auch der Arzt während der Konsultation sofort Zugriff darauf hat. Auch ihre Stammdaten wie Adresse, Telefonnummer und EMail können die Patienten auf diese Weise eingeben oder ändern. Dies trägt auch zu einer höheren Datenqualität bei - das iPad verhindert Fehler, die etwa beim Diktieren oder Buchstabieren solcher Angaben gegenüber dem Praxispersonal auftreten können.

\title{
E-Arztbrief: Ärzte können Förderung ab Januar nutzen
}

Vertragsärzte, die ab Januar 2016 von der Förderung elektronischer Arztbriefe profitieren wollen, werden sich mit den meisten Kollegen bereits verschlüsselt online austauschen können. Das hat ein Test bei der KV Telematik gezeigt.

$\mathrm{D}$ er systemübergreifende Befundaustausch per E-Arztbrief wird bei den meisten Vertragsärzten ab Januar 2016 funktionieren, das hat der Interoperabilitätsworkshop E-Arztbrief von KV Telematik und Bundesverband Gesundheits-IT (bvitg) gezeigt. Damit ist der Weg für Ärzte frei, die von der im EHealth-Gesetz avisierten Förderung des elektronischen Arztbriefes mit 55 Cent je Arztbrief gleich profitieren wollen. Diese Förderung soll laut Gesetzentwurf auch im Januar 2016 starten.

„Unser Workshop hat gezeigt, dass sich die Softwarehersteller auf die gesetzliche Forderung und die Nachfrage der Ärzte vorbereitet haben“, betonte Dr. Florian Fuhrmann, Geschäftsführer der KV Telematik $\mathrm{GmbH}$, eines Tochterunterunternehmens der KBV. Der Workshop mit dem bvitg sei „hervorragend gelaufen“, so Fuhrmann. „Die Interoperabilität zwischen den Systemen kommt voran." 16 Praxis-EDV-Systeme mit einem Marktanteil von $71 \%$ hätten am Workshop teilgenommen und Testarztbriefe gesendet sowie empfangen, weitere neun Systeme mit 5\% Marktanteil seien bereits auditiert. Die Briefe würden über das sichere Netz der KVen versendet und über KV-Connect so verschlüsselt, dass nur der vorgesehene Empfänger den Brief wieder entschlüsseln kann. Das heißt, der E-Arztbrief kann bereits genutzt werden, bevor die Telematikinfrastruktur bereit steht.

„Die beteiligten Unternehmen haben bewiesen, dass sie den E-Arztbrief via KV-Connect professionell umgesetzt haben und die Systeme miteinander interoperabel funktionieren“, erklärte Ekkehard Mittelstaedt, Geschäftsführer des bvitg. Somit könnten die Ärzte, die diese Systeme einsetzen, in vollem Umfang
Die Jenaer Programmierer haben auBerdem die Vergabe von Terminen auf das Tablet übertragen: „Wenn ich den Patienten zum Beispiel für eine Kontrolluntersuchung nach etwa 14 Tagen einbestellen möchte, kann ich dies in der Software anwählen. Die Medizinische Fachangestellte gibt dem Patienten dann das iPad, auf dem eine Reihe freier Termine zur Auswahl steht." Der Patient wählt das für ihn günstigste Datum aus, und seine Entscheidung wird umgehend in den Terminplan der Praxis eingetragen. Auch über eine Maske auf der Webseite der Praxis können Patienten Termine vereinbaren, wovon sie laut Unkelbach zunehmend Gebrauch machen. "Das läuft so rund, dass man sich an die Zeiten von Papierformularen und -kalendern gar nicht mehr erinnern mag", resümiert der Arzt.

Hannes Rügheimer

mit Kollegen elektronisch kommunizieren und auch Befunde austauschen. Bei einigen Systemen ist laut Fuhrmann bereits der Versand strukturierter Arztbriefe möglich, das heißt, die Befunde können direkt in der Patientenkartei elektronisch abgelegt werden. Bei den anderen Systemen ist zumindest der Versand angehängte PDF-Dateien über KV-Connect möglich. Hauke Gerlof

\section{Förderung von E-Arztbriefen}

$\S 291 \mathrm{~h} \mathrm{SGB} \mathrm{V}$, laut Entwurf des E-Health-Gesetzes:

(1) Der Zuschlag nach (...) erhöht sich in den Jahren 2016 und 2017 um eine Pauschale von 55 Cent pro Übermittlung eines elektronischen Briefs zwischen den an der vertragsärztlichen Versorgung teilnehmenden Ärzten und Einrichtungen, wenn die Übermittlung durch sichere elektronische Verfahren erfolgt und dadurch der Versand durch Post-, Boten- oder Kurierdienste entfällt.

Die Systeme, die den E-Arztbrief umgesetzt haben, im Internet: www.kv-telematik.de (unter KV-Connect, Audit-Register) 